\title{
OUTER MEASURES ASSOCIATED WITH LATTICE MEASURES AND THEIR APPLICATION
}

\author{
CHARLES TRAINA \\ St. John's University \\ Department of Mathematics \& Computer Science \\ Jamaica, NY 11439 \\ (Received March 18, 1994 and in revised form July 7, 1994)
}

\begin{abstract}
Consider a set $X$ and a lattice $\mathcal{L}$ of subsets of $X$ such that $\phi, X \in \mathcal{L} . M(\mathcal{L})$ denotes those bounded finitely additive measures on $\mathcal{A}(L)$ which are studied, and $I(L)$ denotes those elements of $M(L)$ which are 0-1 valued. Associated with a $\mu \in M(L)$ or a $\mu \in M_{\mathrm{o}}(L)$ (the elements of $M(L)$ which are $\sigma$-smooth on $L$ ) are outer measures $\mu^{\prime}$ and $\mu^{\prime \prime}$. In terms of these outer measures various regularity properties of $\mu$ can be introduced, and the interplay between regularity, smoothness, and measurability is investigated for both the $0-1$ valued case and the more general case. Certain results for the special case carry over readily to the more general case or with at most a regularity assumption on $\mu^{\prime}$ or $\mu^{\prime \prime}$, while others do not. Also, in the special case of $0-1$ valued measures more refined notions of regularity can be introduced which have no immediate analogues in the general case.
\end{abstract}

KEY WORDS AND PHRASES: Normal lattice, lattice regular measures, associated outer measures.

1991 AMS SUBJECT CLASSIFICATION CODES: 28C15, $28 A 12$.

\section{INTRODUCTION}

Our first aim in this paper is (see Section 3) to obtain further properties of two outer measures $\mu^{\prime}$ and $\mu^{\prime \prime}$ (see below for definitions) associated first with $\mu \in I(L)$ and $\mu \in I_{\mathrm{o}}(L)$ and subsequently with $\mu \in M(L)$ and $\mu \in M_{\mathrm{o}}(L)$, and to apply these properties to characterize various classes of measures. In the former case we thereby extend results of $[6,7,8]$. Also in the case of $\mu \in I_{\sigma}(L)$ we consider in further detail the subset $I_{s}(\mathcal{L})$ of $I_{\mathrm{o}}(L)$ of slightly regular measures (see below for definition) and hence extend the work of [6].

We note that in the case of $0-1$ valued measures such as $\mu \in I_{\mathrm{o}}(\mathcal{L})$, the associated outer measure $\mu^{\prime \prime}$ is clearly regular and $S_{\mu^{\prime \prime}}$ the $\mu^{\prime \prime}$-measurable sets can be explicitly characterized. This is no longer the case if $\mu \in M_{\mathrm{o}}(L)$ and we must hypothesize regularity of $\mu^{\prime \prime}$ in certain cases in order to generalize the two-valued case. Also in general the characterization of $S_{\mu^{\prime \prime}}$ is not as explicit as in the two-valued case, and this further complicates the general situation. If $\mu \in J(L)$, i.e., is strongly $\sigma$-smooth (see below) and if $\mathcal{L}$ is a $\delta$-lattice then $\mu^{\prime}=\mu^{\prime \prime}$ and, hence, $S_{\mu^{\prime}}=S_{\mu^{\prime \prime}}$, and $S_{\mu^{\prime}}$, has been characterized explicitly in [5]. We recall this result in Section 4 and build on it to extend some of the results in [5], see in particular Theorems 4.2, 4.3, 4.4.

We begin with a review of the notations (section 2) which will be used throughout the paper, as well as a review of the relevant definitions needed. Further related matters can be found in $[1,2,3]$.

\section{BACKGROUND AND NOTATION}

We introduce the necessary measure theoretic, and lattice definitions, and note the known properties about lattice measures that we shall need.

The definitions and notations are standard and are consistent with those found in, for example, $[1,3,9]$. We collect the ones we need and some of their properties for the reader's convenience. 
Let $X \neq \varnothing$ be an abstract set, $L$ a lattice of subsets of $X$, which we will assume throughout. We shall assume that $\varnothing, X \in \mathcal{L}$. For $E \subset X, E^{\prime}$ denotes its complement. We denote by:

(1) $\mathcal{A}(\mathcal{L})$, the algebra generated by $\mathcal{L}$;

(2) $\delta(L)$, the lattice of all countable intersections of sets from $L$;

(3) $L^{\prime}$, the lattice of complements of sets from $L$.

We introduce the following measure theoretic definitions.

Def ${ }^{n}$ : The set of all nonnegative finite valued, finitely additive (f.a.), bounded measures on $\mathcal{A}(\mathcal{L})$ will be denoted by $M(L)$.

An element $\mu \in M(\mathcal{L})$ is said to be $\sigma$-smooth on $\mathcal{L}$ iff whenever $L_{n} \in \mathcal{L}, n=1,2, \ldots$, and $L_{n} \downarrow \varnothing$, then $\mu\left(L_{n}\right) \rightarrow 0$.

An element $\mu \in M(L)$ is said to be $\sigma$-smooth on $\mathcal{A}(L)$ iff whenever $A_{n} \in \mathcal{A}(L), n=1,2, \ldots$, and $A_{n} \downarrow \varnothing$, then $\mu\left(A_{n}\right) \rightarrow 0$. (Note that this condition is equivalent to $\mu$ being countably additive.)

An element $\mu \in M(\mathcal{L})$ is said to be strongly $\sigma$-smooth on $\mathcal{L}$ iff whenever $L, L_{n} \in \mathcal{L}, n=1,2, \ldots$, and $L_{n} \downarrow L, L=\bigcap_{n=1}^{\infty} L_{n}$, then $\mu(L)=\inf \left\{\mu\left(L_{n}\right) \mid n=1,2, \ldots\right\}$.

An element $\mu \in M(L)$ is said to be $L$-regular iff for any $A \in \mathcal{A}(L), \mu(A)$ $\sup \{\mu(L) \mid L \subset A, L \in L\}$.

The following notation is used to denote the subsets of $M(L)$ determined by the above properties: $M_{\mathrm{o}}(L)$ is the set of measures that are $\sigma$-smooth on $L$;

$M^{\mathcal{G}}(\mathcal{L})$ is the set of measures that are o-smooth on $\mathcal{A}(\mathcal{L})$;

$J(L)$ is the set of measures that are strongly o-smooth on $L$;

$M_{R}(L)$ is the set of $L$-regular measures;

$M_{R}^{\sigma}(L)$ is the set of $L$-regular measures of $M^{\circ}(L)$.

We note that $J(L) \subset M_{\mathrm{o}}(L)$, and $M_{R}(L) \cap M_{\mathrm{o}}(L) \subset M^{\mathrm{\sigma}}(\mathcal{L})$.

We denote by $I(L), I_{\mathrm{o}}(\mathcal{L}), \Phi(L), I_{R}(\mathcal{L})$, and $I_{R}^{\mathrm{\sigma}}(\mathcal{L})$ the subsets of the corresponding $M$ 's that consist of the non-trivial 0-1 valued measures. We shall write $\mu \leq \nu(L)$ whenever $\mu, v$ are measures, or set functions such that $\mu(L) \leq \nu(L)$ for all $L \in L$.

Observe the following enlargement, i.e., with each $\mu \in I(L)$ there is a $v \in I_{R}(L)$ s.t. $\mu \leq v(L)$; and for each $\mu \in M(\mathcal{L})$ there is a $v \in M_{R}(L)$ s.t. $\mu \leq v(\mathcal{L})$ and $\mu(X)=v(X)$.

For these results and other related matters see $[5,7,8]$. If $\mu \in M(L)$, we define a set function $\mu^{\prime}$ on $X$ by: For $E \subset X, \mu^{\prime}(E)=\inf \left\{u\left(L^{\prime}\right) \mid E \subset L^{\prime}, L \in \mathcal{L}\right\}$. The function $\mu^{\prime}$ has the following properties:

(1) For every $E \subset X, 0 \leq \mu^{\prime}(E)<+\infty$,

(2) $\mu^{\prime}(\varnothing)=0$,

(3) If $E \subset F$, then $\mu^{\prime}(E) \leq \mu^{\prime}(F)$,

(4) $\mu \leq \mu^{\prime}(L)$,

(5) $\mu=\mu^{\prime}$ on $\mathcal{L}$ iff $\mu \in M_{R}(\mathcal{L})$,

(6) $\mu=\mu^{\prime}$ on $\mathcal{L}^{\prime}$,

If $\mu \in M_{\sigma}(L)$, we define a set function $\mu^{\prime \prime}$ on $X$ by: For $E \subset X, \mu^{\prime \prime}(E)=$ $\inf \left\{\sum_{i=1}^{\infty} \mu\left(L_{i}^{\prime}\right) \mid E \subset \bigcup_{i=1}^{\infty} L^{\prime}{ }_{i}, L_{i} \in L, i=1,2, \ldots\right\} \cdot \mu^{\prime \prime}$ is in fact an outer measure. We note that in the case of $0-1$ measures, if $\mu \notin I_{\sigma}(L)$, then $\mu^{\prime \prime}=0$. 
For the $\mathcal{L}$-regular measures the following holds:

(1) $\mu \in M_{R}(L)$ iff $\mu \in M(L)$ and $\mu\left(L^{\prime}\right)=\sup \left\{\mu(K) \mid K \subset L^{\prime}, K \in \mathcal{L}\right\}$ for every $L \in \mathcal{L}$.

(2) $\mu \in M_{R}(L)$ iff $\mu \in M(L)$ and $\mu(A)=\inf \left\{\mu\left(L^{\prime}\right) \mid A \subset L^{\prime}, L \in L\right\}$ for every $A \in \mathcal{A}(L)$.

The function $\mu^{\prime}$ gives rise to another set of measures in $M(L)$.

Def $^{n}$ : An element $\mu \in M(L)$ is said to be weakly regular iff for every $L \in \mathcal{L}$,

$$
\mu\left(L^{\prime}\right)=\sup \left\{\mu^{\prime}(K) \mid K \subset L^{\prime}, K \in L\right\} .
$$

The set of weakly regular measures will be denoted by $M_{w}(L)$, and the corresponding subset of $0-1$ measures by $I_{w}(L)$.

We now recall some lattice definitions:

Def $^{n}$ : (a) A lattice $\mathcal{L}$ is said to be normal iff for any $A, B \in \mathcal{L}$ s.t. $A \cap B=\varnothing$ there exists $C, D \in \mathcal{L}$ s.t. $A \subset C^{\prime}, B \subset D^{\prime}$ and $C^{\prime} \cap D^{\prime}=\varnothing$.

(2) A lattice $\mathcal{L}$ is said to be a delta-lattice ( $\delta$-lattice) iff $\mathcal{L}=\delta(L)$.

(3) A lattice $L$ is said to be complement generated (c.g.) iff for $L \in \mathcal{L}$, there is a sequence $L_{n} \in \mathcal{L}, n=1,2, \ldots$ s.t. $L=\bigcap_{n=1}^{\infty} L_{n}^{\prime}$.

(4) $L$ is said to be countably paracompact (c.p.) iff for any sequence $\left\{A_{n}\right\}$ from $\mathcal{L}$ s.t. $A_{n} \downarrow \varnothing$, there exists a sequence $\left\{B_{n}\right\}$ from $\mathcal{L}$ s.t. $A_{n} \subset B^{\prime}{ }_{n}, n=1,2, \ldots$, and $B^{\prime}{ }_{n} \downarrow \varnothing$.

(5) If $L_{1}$ and $L_{2}$ are two lattices of subsets of $X$, then $L_{1}$ semi-separates $L_{2}\left(L_{1}\right.$ s.s. $\left.L_{2}\right)$ iff $A \in L_{1}$, $B \in L_{2}$ and $A \cap B=\varnothing$ implies there exists $C \in L_{1}, B \subset C$, and $A \cap C=\phi$.

(6) If $\mathcal{L}_{1}$ and $\mathcal{L}_{2}$ are two lattices of subsets of $X$, then $\mathcal{L}_{1}$ separates $\mathcal{L}_{2}$ iff $A, B \in \mathcal{L}_{2}$ and $A \cap B=\phi$ implies there exists $C, D \in L_{1}$ s.t. $A \subset C, B \subset D$ and $C \cap D=\phi$.

(7) $L$ is complemented iff $L \in \mathcal{L}$ implies $L^{\prime} \in \mathcal{L}$ (i.e., $\mathcal{L}$ is an algebra).

(8) $\mathcal{L}$ is countably compact (c.c.) iff for every sequence $\left\{L_{n}\right\}$ from $\mathcal{L}$ s.t. $\bigcap_{n=1}^{\infty} L_{n}=\varnothing$, then there exists $L_{n_{1}}, L_{n_{2}}, \ldots, L_{n_{k}}$ s.t. $\bigcap_{i=1}^{k} L_{n_{i}}=\varnothing$.

We note that normality of a lattice has the following equivalent formulations:

(a) $L$ is normal iff $\mu \in I(L), \mu \leq v_{1}, \mu \leq v_{2}, v_{1}, v_{2} \in I_{R}(L)$ then $v_{1}=v_{2}$.

(b) $L$ is normal iff $\mu \in I(L), \nu \in I_{R}(L)$ and $\mu \leq \nu(L)$, then $\mu \leq v=\nu^{\prime}=\mu^{\prime}$ on $L$.

(c) $L$ is normal iff whenever $L \subset L^{\prime}{ }_{1} U L^{\prime}{ }_{2}$ where $L, L_{1}, L_{2} \in \mathcal{L}$ then $L=A \cup B$ where $A, B \in \mathcal{L}$ and $A \subset L^{\prime}, B \subset L^{\prime}{ }_{2}$.

We also list some further consequences of normality as well as some relations involving the already noted sets of measures. Further details can be found in $[6,7,8]$ as well as below. If $\mathcal{L}$ is a $\delta$-lattice, $\mu \in J(L)$, then $\mu\left(\bigcup_{i=1}^{\infty} L^{\prime}{ }_{i}\right) \leq \sum_{i=1}^{\infty} \mu\left(L_{i}^{\prime}\right)$,

$$
L_{i} \in \mathcal{L}, \quad i=1,2, \ldots .
$$

If $\mu \in M_{\mathrm{o}}(L)$, then $\mu(X)-\mu^{\prime \prime}(X)$ and $\mu \leq \mu^{\prime \prime}(L)$.

If $\mathcal{L}$ is c.g. and $\mu \in M_{\mathrm{\sigma}}\left(\mathcal{L}^{\prime}\right)$, then $\mu \in M_{R}(\mathcal{L})$.

If $\mathcal{L}$ is c.g. and normal, and $\mu \in J(\mathcal{L})$, then $\mu \in M_{R}^{\mathrm{o}}(\mathcal{L})$.

If $\mathcal{L}$ is normal then $M_{w}(\mathcal{L})=M_{R}(\mathcal{L})$.

(2.6) If $\mu \in I(L)$ then $S_{\mu^{\prime}}=\left\{E \subset X \mid E \supset L, \mu(L)-1, L \in \mathcal{L}\right.$; or $\left.E^{\prime} \supset L, \mu(L)=1, L \in \mathcal{L}\right\}$, 
(the set of $\mu^{\prime}$-measurable subsets of $X$ ). Consequently, $L \subset S_{\mu^{\prime}}$ iff $\mu \in I_{R}(L)$.

$$
\text { If } \mu \in I_{\mathrm{o}}(\mathcal{L}) \text {, then }
$$

$S_{\mu^{\prime \prime}}=\left\{E \subset X \mid E \supset \bigcap_{n=1}^{\infty} L_{n}, L_{n} \in \mathcal{L}, \mu\left(L_{n}\right)=1, n=1,2, \ldots\right.$; or $\left.E^{\prime} \supset \bigcap_{n=1}^{\infty} L_{n}, L_{n} \in \mathcal{L}, \mu\left(L_{n}\right)=1, n=1,2, \ldots\right\}$, (the set of $\mu^{\prime \prime}$ measurable subsets of $X$ ).

We note that $S_{\mu^{\prime}} \subset S_{\mu^{\prime \prime}}$ if $\mu \in I_{\sigma}(\mathcal{L})$.

\section{Note:}

(1) The converse condition of (2.5) is false in the following sense: $I_{w}(L)=I_{R}(L)$ does not imply $\mathcal{L}$ is normal.

\section{Counterexample:}

Let $X \neq \varnothing$ and let $A, B \subset X$ s.t. $A \cap B=\varnothing, A \cup B \neq X$. Let $\mathcal{L}=\{\varnothing, X, A, B, A \cup B\}$. Then, $L$ is a lattice that is not normal, but $I_{w}(L)=I_{R}(L)$.

(2) We note the inequality, $\Phi(L) \neq I_{\mathrm{\sigma}}(\mathcal{L})$.

\section{Counterexample:}

Let $X \approx \varnothing$ be a set, $\mathcal{L}$ a lattice of subsets of $X$. If $\mathcal{L}$ is c.g. and normal, then $\Phi(L)=I_{R}^{\circ}(\mathcal{L})$, and if $\mathcal{L}$ is c.c. then $I(L)=I_{\mathrm{\sigma}}(L)$.

Therefore, if $I_{\mathrm{o}}(L)=\Phi(L)$, then we have:

$$
I(L)=I_{\sigma}(L)=\Phi(L)=I_{R}^{\mathrm{g}}(\mathcal{L})-I_{R}(L) .
$$

$\Rightarrow \mathcal{L}$ is complemented. Now, take $(X, G)$ to be a $T_{31 / 2}$ topological space. Let $\mathcal{L}=Z$, the zero sets, i.e., for each continuous real-valued function, $f$, on $X, Z(f)=\{x \in X \mid f(x)=0\}$.

We choose $Z$ so that it is not an algebra. Now $Z$ is c.g. and normal. If we let $X$ be pseudocompact so that $I_{\mathrm{o}}(Z)=I(Z)$, see [4], then there is a $\mu \in I_{\mathrm{o}}(Z)$ s.t. $\mu \notin \Phi(Z)$.

\section{SOME FURTHER RESULTS ON 0-1 VALUED LATTICE MEASURES}

There are several relations that exist between the 0-1 lattice measures that can hold when certain conditions are imposed on the underlying lattice of subsets. In this section we shall consider such relations.

THEOREM 3.1. Let $X \neq \varnothing$ be a set, $\mathcal{L}$ a lattice of subsets.

(a) If $\mathcal{L}$ is normal, $\mu \in I_{\mathrm{\sigma}}(\mathcal{L}), v \in I_{R}(\mathcal{L})$, and $\mu \leq \nu(\mathcal{L})$, then $v \in I_{\mathrm{\sigma}}\left(\mathcal{L}^{\prime}\right)$.

(b) If $\mathcal{L}$ is $\delta$-normal, $\mu \in I_{\mathrm{o}}(\mathcal{L}), v \in I_{R}(\mathcal{L})$, and $\mu \leq \nu(L)$, then $v \in \Phi\left(\mathcal{L}^{\prime}\right)$.

(c) If $L$ is normal, $\mathcal{L}$ s.s. $\delta(L), \mu \in \Phi(L), v \in I_{R}(L), \mu \leq v(L)$, then $v \in \Phi\left(L^{\prime}\right)$.

PROOF. We refer to [7].

We consider next $I_{s}(\mathcal{L})$, introduced in [6]. We recall that $\mu \in I_{s}(\mathcal{L})$ iff $\mu \in I_{\mathrm{\sigma}}(\mathcal{L})$ and whenever $L \in \mathcal{L}$ s.t. $\mu\left(L^{\prime}\right)=1$, then there exists $L_{n} \in \mathcal{L}$ s.t. $L^{\prime} \supset \bigcap_{n=1}^{\infty} L_{n}$ and $\mu\left(L_{n}\right)=1$ for $n=1,2, \ldots$. We obtain some further characterizations of $I_{s}(\mathcal{L})$, some new and some known, but in alternate ways.

We first note the following:

PROPOSITION 3.1. If $\mu \in I_{s}(L)$, then $\mu \in I^{\sigma}(L)$ and $L \subset S_{\mu^{\prime \prime}}$.

PROOF. We always have $\mu \leq \mu^{\prime \prime}$ on $\mathcal{L}$, since $\mu \in I_{\mathrm{s}}(\mathcal{L})$. (Recall: If $\mu \notin I_{\mathrm{\sigma}}(\mathcal{L})$, then $\mu^{\prime \prime}=0$.)

Suppose that $\mu(L)=0$ for $L \in \mathcal{L}$. Then $\mu\left(L^{\prime}\right)=1$, and since $\mu \in I_{s}(\mathcal{L}), L^{\prime} \supset \bigcap_{n=1}^{\infty} L_{n}$, where $L_{n} \in \mathcal{L}, \mu\left(L_{n}\right)=1$ for $n=1,2, \ldots$. Therefore, $L \subset \cup_{n=1}^{\infty} L^{\prime}{ }_{n}, L_{n} \in \mathcal{L}, \mu\left(L^{\prime}{ }_{n}\right)=0$ for $n=1,2, \ldots$. There- 
fore, $\mu^{\prime \prime}(L)=0$ and so $\mu=\mu^{\prime \prime}$ on $L$.

Now let $L \in \mathcal{L}$ s.t. $\mu\left(L^{\prime}\right)=0$. Then $\mu^{\prime \prime}\left(L^{\prime}\right)-0\left(\right.$ since $\mu^{\prime \prime} \leq \mu$ on $\left.L^{\prime}\right)$, and so $L^{\prime} \in S_{\mu^{\prime \prime}}$, hence $L \in S_{\mu^{\prime \prime}}$.

If $\mu\left(L^{\prime}\right)-1$, then since $\mu \in I_{s}(L), L^{\prime} \supset \bigcap_{n=1}^{\infty} L_{n}, L_{n} \in \mathcal{L}, \mu\left(L_{n}\right)-1$ for $n-1,2, \ldots$. Therefore by 2.7 of Section 2, $L^{\prime} \in S_{\mu^{\prime \prime}}$, hence $L \in S_{\mu^{\prime \prime}}$. Therefore, $L \subset S_{\mu^{\prime \prime}}$, whence $\mathcal{A}(L) \subset S_{\mu^{\prime \prime}}$, and so $\mu=\mu^{\prime \prime}$ on $L$. Therefore $\mu \in I^{\sigma}(L)$ since $\mu^{\prime \prime}$ is an outer measure.

The proof is now complete.

PROPOSITION 3.2. If $\mu \in I_{s}(L)$ and if $L$ s.s. $\delta(L)$, then $\mu \in I_{R}^{\sigma}(L)$.

PROOF. By Proposition 3.1, one need only show that $\mu \in I_{R}(L)$. Let $L \in L$.

(i) If $\mu\left(L^{\prime}\right)=0$, the monotonicity of $\mu$ shows that $\sup \left\{\mu(K) \mid K \subset L^{\prime}, K \in L\right\}=0$.

(ii) If $\mu\left(L^{\prime}\right)=1$, then since $\mu \in I_{s}(L)$, there exists a sequence $\left\{L_{n}\right\}$ from $L$ s.t. $L^{\prime} \supset \bigcap_{n=1}^{\infty} L_{n}$ and $\mu\left(L_{n}\right)=1, n-1,2, \ldots$ Let $B=\bigcap_{n=1}^{\infty} L_{n} \in \delta(L)$. Then $L \cap B=\varnothing$, and since $L$ s.s. $\delta(L)$ there exists $K_{0} \in L$ s.t. $B \subset K_{0}$ and $L \cap K_{0}=\varnothing$, so $K_{0} \subset L^{\prime}$. Since $\mu$ is finitely additive, and $X=L^{\prime} \cup K_{0}^{\prime}, \mu\left(K_{0}^{\prime}\right)=0 \Rightarrow \mu\left(K_{0}\right)=1$. Therefore $\sup \left\{\mu(K) \mid K \subset L^{\prime}, K \in L\right\}=1$.

Hence, for any $L \in L, \mu\left(L^{\prime}\right)=\sup \left\{\mu(K) \mid K \subset L^{\prime}, K \in \mathcal{L}\right\}$, and so $\mu \in I_{R}(L)$. Therefore, $\mu \in I_{R}^{\mathrm{o}}(\mathcal{L})$.

The proof is now complete.

PROPOSITION 3.3. If $\mu \in \Phi(L)$, then $\mu$ can be extended uniquely to a $v \in \Phi(\delta(L)$ ). (The proof is omitted.)

We now give some alternate characterizations of the measures in $I_{s}(\mathcal{L})$.

THEOREM 3.2. $\mu \in I_{S}(L)$ iff $\mu=\lambda$, where $\lambda \in I_{R}^{\mathrm{o}}(\delta(L)$ ) (where $\lambda \mid$ is the restriction of $\lambda$ to $\mathcal{A}(L))$.

PROOF. Assume that $\mu \in I_{s}(L)$. Then $\mu \in \Phi(L)$, and so by Proposition 3.3, we can extend $\mu$ uniquely to a $\lambda \in \Phi(\delta(L))$, defined by: For $A=\bigcap_{n=1}^{\infty} L_{n} \in \delta(L)$, where $L_{n} \downarrow A, \lambda(A)=$ $\inf \left\{\mu\left(L_{n}\right) \mid n-1,2, \ldots\right\}$.

Let $D=\bigcap_{n=1}^{\infty} L_{n} \in \delta(L)$ and suppose that $L\left(D^{\prime}\right)=1$. Then

$$
\lambda\left(\bigcup_{n=1}^{\infty} L_{n}^{\prime}\right)=1
$$

Therefore,

$$
\lambda\left(\bigcup_{n=1}^{\infty} L_{n}^{\prime}\right) \leq \sum_{n=1}^{\infty} \lambda\left(L_{n}^{\prime}\right) \text { by } 2.1 .
$$

It follows from (1) and (2) that $\lambda\left(L^{\prime}{ }_{n}\right)=1$ for some $n \in N$. Since $\mu-\lambda \mid, \mu\left(L_{n}^{\prime}\right)-1$. But $\mu \in I_{s}(L)$ and so $L^{\prime}{ }_{n} \supset \bigcap_{m=1}^{\infty} K_{m}, K_{m} \in \mathcal{L}, \mu\left(K_{m}\right)=1$ for $m=1,2, \ldots$. Therefore, $\lambda\left(\bigcap_{m=1}^{\infty} K_{m}\right)=1 \Rightarrow \lambda \in I_{R}^{\sigma}(\delta(L))$. The converse is clear. The proof is now complete.

THEOREM 3.3. Let $\mu \in I_{\mathrm{o}}(\mathcal{L})$. Then

(1) $\mu=\mu^{\prime \prime}$ on $\mathcal{L}$ iff $\mu \in I_{s}(\mathcal{L})$.

(2) If $\mu-\mu^{\prime \prime}$ on $\mathcal{L}$, then $L \subset S_{\mu^{\prime \prime}}$ and $\mu \in I^{\circ}(\mathcal{L})$. 
PROOF. (1) Suppose $\mu-\mu^{\prime \prime}$ on $L$.

Let $L \in \mathcal{L}$ s.t. $\mu\left(L^{\prime}\right)-1$. Then $\mu(L)=0-\mu^{\prime \prime}(L)$. Hence, there exists $K_{n} \in L, n=1,2, \ldots$ s.t. $L \subset \cup_{n-1}^{\infty} K^{\prime}{ }_{n}$ and $\mu\left(K_{n}^{\prime}\right)=0$ for $n=1,2, \ldots$. Therefore, $L^{\prime} \supset \bigcap_{n=1}^{\infty} K_{n}$ where $K_{n} \in \mathcal{L}, \mu\left(K_{n}\right)-1$ for $n=1,2, \ldots$. Therefore, $\mu \in I_{s}(\mathcal{L})$.

For the converse, suppose $\mu \in I_{s}(L)$.

Since $\mu \leq \mu^{\prime \prime}$ on $L$, we need only consider the case when $L \in \mathcal{L}$ s.t. $\mu(L)=0$. Then, $\mu\left(L^{\prime}\right)=1$ and since $\mu \in I_{s}(\mathcal{L})$, there exists $K_{n} \in \mathcal{L}, n-1,2, \ldots$ s.t. $L^{\prime} \supset \bigcap_{n=1}^{\infty} K_{n}$ and $\mu\left(K_{n}\right)-1$ for $n-1,2, \ldots$. Therefore, $L \subset \bigcap_{n=1}^{\infty} K_{n}^{\prime}$ with $\mu\left(K_{n}^{\prime}\right)=0$ for $n=1,2, \ldots$. Therefore, $\sum_{n-1}^{\infty} \mu\left(K_{n}^{\prime}\right)=0 \Rightarrow \mu^{\prime \prime}(L)-0$. Hence, $\mu-\mu^{\prime \prime}$ on $L$.

(2) is immediate since $\mu=\mu^{\prime \prime}$ on $\mathcal{L}$ implies $\mu \in I_{s}(L)$ by part (1) and the result now follows by Proposition 3.1.

The proof is now complete.

Note:

$$
\mu \in \Phi(L) \text {, iff } \mu^{\prime}=\mu=\mu^{\prime \prime} \text { on } L^{\prime} \text {. }
$$

THEOREM 3.4. Let $\mu \in \Phi(\mathcal{L}), v \in I_{\mathrm{o}}(\mathcal{L}), \mu \leq \nu(\mathcal{L})$, and $S_{\mu^{\prime \prime}} \cap \mathcal{L}=S_{v^{\prime \prime}} \cap \mathcal{L}$. Then $v \in \Phi(\mathcal{L})$.

PROOF. Assume that $v \notin \Phi(L)$. Then there exists $L_{0} \in \mathcal{L}$ and a sequence $\left\{L_{n}\right\}$ from $L$ s.t. $L_{n} \downarrow L_{0}, L_{0}=\bigcap_{n=1}^{\infty} L_{n}$, but $v\left(L_{0}\right) \neq \inf \left\{v\left(L_{n}\right) \mid n=1,2, \ldots\right\}$. Since $v$ is a $0-1$ measure, and monotonic,

(1) $v\left(L_{0}\right)=0$ and $v\left(L_{n}\right)=1$ for $n=1,2, \ldots$

(2) Now, $\mu \leq \nu(L)$, so $\mu\left(L_{0}\right)=0$, hence $\mu\left(L_{0}^{\prime}\right)=1$.

Since $L_{0}=\bigcap_{n=1}^{\infty} L_{n}$, with $L_{n} \in \mathcal{L}, v\left(L_{n}\right)=1$ for $n=1,2, \ldots$, it follows from 2.7 that $L_{0} \in S_{v^{\prime \prime}}=S_{\mu^{\prime \prime}}$. But, $\mu \leq v \leq v^{\prime \prime} \leq \mu^{\prime \prime}$ on $\mathcal{L}$ and $v^{\prime \prime} \leq v=v^{\prime} \leq \mu^{\prime}=\mu^{\prime \prime}$ on $\mathcal{L}^{\prime}$, with $\mu^{\prime}=\mu^{\prime \prime}$ on $\mathcal{L}^{\prime}$ since $\mu \in \Phi(\mathcal{L})$.

Now $v^{\prime \prime}\left(L_{n}\right)=1$ for all $n$ since $v\left(L_{n}\right)=1$ for all $n$, and $v^{\prime \prime}\left(L_{0}\right)=1$, since $v^{\prime \prime}\left(L^{\prime}\right)=0$. Therefore, $\mu^{\prime \prime}\left(L_{0}\right)=1$.

Now $\mu\left(L_{0}\right)=0$, so there exists $N \in \mathrm{N}$ s.t. $\mu\left(L_{n}\right)=0$ for all $n \geq N$, since $\mu \in \Phi(L)$. We have $L^{\prime}{ }_{n} \uparrow L_{0}^{\prime}$, so $\mu\left(L_{n}^{\prime}\right)=1$ for $n \geq N$. Therefore $\mu^{\prime \prime}\left(L_{n}^{\prime}\right)=1$ for $n \geq N$, and so $\mu^{\prime \prime}\left(L_{0}^{\prime}\right)-1$ since $\mu^{\prime \prime}$ is a regular outer measure. But $\mu^{\prime \prime}\left(L_{0}\right)=1$, therefore $L_{0} \notin S_{\mu^{\prime \prime}}$, a contradiction. Therefore it must be that $v \in \Phi(L)$.

The proof is now complete.

THEOREM 3.5. Suppose that $\mu \in I_{s}(L), v \in \Phi(L), \mu \leq v(L)$. Then $\mu=v$.

PROOF. Since $\mu \in I_{s}(L)$, then by Theorem 3.2, $\mu=\lambda \mid$ where $\lambda \in I_{R}^{\mathrm{o}}(\delta(L))$, and $\lambda\left(\bigcap_{n=1}^{\infty} L_{n}\right)-\inf \left\{\mu\left(L_{n}\right) \mid L_{n} \in \mathcal{L}, n=1,2, \ldots\right\}$. By Proposition 3.3, $v$ can be extended to a $\hat{v} \in \Phi(\delta(L))$ where

$$
\hat{v}\left(\bigcap_{n=1}^{\infty} L_{n}\right)=\inf \left\{v\left(L_{n}\right) \mid L_{n} \in \mathcal{L}, n=1,2, \ldots\right\} .
$$

Since $\mu \leq \nu(L), \lambda \leq \hat{v}$ on $\delta(L)$. Therefore $\lambda=\hat{v} \Rightarrow \mu=\nu$ on $\mathcal{A}(L)$.

The proof is now complete. 


\section{THE GENERAL CASE $M(L)$}

In this section we consider the non $0-1$ measures on $\mathcal{A}(\mathcal{L})$. In particular, we obtain results pertaining to regular outer measures, and results which insure that certain elements of $M(L)$ are regular.

Def $^{n}$ : Let $X \neq \not$ be a set, $L$ a lattice of subsets of $X$. Let $\mu \in M(L)$. For $E \subset X$ we define: $\mu_{\iota}(E)=\sup \{\mu(L) \mid L \subset E, L \in \mathcal{L}\}$.

We note that the set functions $\mu^{\prime}$ and $\mu_{t}$ have the following relations:

(1) $\mu(X)=\mu_{t}\left(E^{\prime}(E)+\mu^{\prime}(E)\right.$ for any $E \subset X$.

(2) If $E \subset X$, then $E \in S_{\mu^{\prime}}$ iff $\mu^{\prime}(E)=\mu_{t}(E)$.

We add the proof of (2) for completeness.

PROOF OF (2): See also [5].

Let $E \in S_{\mu^{\prime}}$. Then

(1) $\mu^{\prime}(X)=\mu(X)=\mu^{\prime}(E)+\mu^{\prime}\left(E^{\prime}\right)$. It follows from (1) and Remark (1) preceding, that

(2) $\mu^{\prime}\left(E^{\prime}\right)=\mu_{t}\left(E^{\prime}\right)$.

Now, using standard arguments involving supremum and infimum, it follows from (2) that $\mu_{i}(E)=\mu^{\prime}(E)$.

Conversely, to show that $E \in S_{\mu^{\prime}}$, it will suffice to show:

$$
\mu^{\prime}\left(A^{\prime}\right) \geq \mu^{\prime}\left(A^{\prime} \cap E\right)+\mu^{\prime}\left(A^{\prime} \cap E^{\prime}\right) \text { where } A \in \mathcal{L} .
$$

Let $\varepsilon>0$ be given and arbitrary. There exists $L \in \mathcal{L}$ s.t. $E \subset L^{\prime}$ and

(1) $\mu\left(L^{\prime}\right)<\mu^{\prime}(E)+\frac{\varepsilon}{2}$

Similarly, there exists $K \in \mathcal{L}$ s.t. $K \subset E$ and

(2) $\mu_{i}(E)-\frac{\varepsilon}{2}<\mu(K)$

Since $K \subset E \subset L^{\prime}$ and $\mu$ is subtractive,

(3) $\mu\left(L^{\prime}-K\right)=\mu\left(L^{\prime}\right)-\mu(K)$

It follows from (1), (2), (3) and the hypothesis on $E$ that

(4) $\mu\left(L^{\prime}-K\right)<\varepsilon$

Let $A^{\prime} \in \mathcal{L}^{\prime}$, and write the disjoint union

$$
A^{\prime} \cap L^{\prime}=\left[A^{\prime} \cap\left(L^{\prime}-K\right)\right] \cup\left(A^{\prime} \cap K\right) .
$$

Since $\mu$ is additive,

(5) $\mu\left(A^{\prime} \cap L^{\prime}\right)=\mu\left(\left[A^{\prime} \cap\left(L^{\prime}-K\right)\right]\right)+\mu\left(A^{\prime} \cap K\right)$

By monotonicity of $\mu$, and (5) and (4) we obtain:

(*) $\mu\left(A^{\prime} \cap L^{\prime}\right)<\mu\left(A^{\prime} \cap K\right)+\varepsilon$

We have $A^{\prime} \cap E \subset A^{\prime} \cap L^{\prime}, A^{\prime} \cap E^{\prime} \subset A^{\prime} \cap K^{\prime}$, and again by monotonicity of $\mu$,

(6) $\mu^{\prime}\left(A^{\prime} \cap E\right)+\mu^{\prime}\left(A^{\prime} \cap E^{\prime}\right) \leq \mu^{\prime}\left(A^{\prime} \cap L^{\prime}\right)+\mu^{\prime}\left(A^{\prime} \cap K^{\prime}\right)$. Since $\mu-\mu^{\prime}$ on $L^{\prime}$, we have

$\mu^{\prime}\left(A^{\prime} \cap L^{\prime}\right)=\mu\left(A^{\prime} \cap L^{\prime}\right), \mu^{\prime}\left(A^{\prime} \cap K^{\prime}\right)=\mu\left(A^{\prime} \cap K^{\prime}\right)$, and so from (6) we obtain

(7) $\mu^{\prime}\left(A^{\prime} \cap E\right)+\mu^{\prime}\left(A^{\prime} \cap E^{\prime}\right) \leq \mu\left(A^{\prime} \cap L^{\prime}\right)+\mu\left(A^{\prime} \cap K^{\prime}\right)$.

By $\left(^{*}\right)$ we obtain from (7):

(8) $\mu^{\prime}\left(A^{\prime} \cap E\right)+\mu^{\prime}\left(A^{\prime} \cap E^{\prime}\right)<\mu\left(A^{\prime} \cap K\right)+\mu\left(A^{\prime} \cap K^{\prime}\right)+\varepsilon$

But, $\mu$ is additive, and $A^{\prime}=A^{\prime} \cap\left(K \cup K^{\prime}\right)$, so we obtain from (8), 
(9) $\mu^{\prime}\left(A^{\prime} \cap E\right)+\mu^{\prime}\left(A^{\prime} \cap E^{\prime}\right)<\mu\left(A^{\prime}\right)+\varepsilon$.

Since $\varepsilon>$ was arbitrary, and $\mu=\mu^{\prime}$ on $L^{\prime}$, we conclude from (9) that

$\mu^{\prime}\left(A^{\prime} \cap E+\mu^{\prime}\left(A^{\prime} \cap E^{\prime}\right)<\mu^{\prime}\left(A^{\prime}\right)\right.$ for any $A^{\prime} \in L^{\prime}$.

Hence, $E \in S_{\mu^{\prime}}$, and the proof is now somplete.

Def $^{n}:$ Let $X \neq \varnothing$ be a set, and let $v$ be a finite, finitely subadditive outer measure defined for all $A \subset X$. Let $S_{v}=\left\{E \subset X \mid v(A) \geq v(A \cap E)+v\left(A \cap E^{\prime}\right)\right.$, for all $\left.A \subset X\right\}$ be the set of all $v$-measurable subsets of $X$. We define a set function $v^{0}$ by: For $E \subset X, v^{0}(E)=\inf \left\{v(M) \mid E \subset M, M \in S_{v}\right\}$.

It follows that $v^{0}$ is itself a finite, finitely subadditive (f.s.a) outer measure s.t. $v^{0}(X)-v(X)$ and $v \leq v^{0}$ for all $E \subset X$.

Def $^{n}$ : Let $X \neq \varnothing$ be a set, $v$ a finite, f.s.a outer measure defined for all subsets of $X$. Let $S_{v}$ be the set of $v$-measurable subsets of $X$.

(1) We say that $v$ is cover regular iff for $A \subset X$ there exists $M \in S_{v}$ s.t. $A \subset M$ and $v(M)=v(A)$.

(2) We say that $v$ is a regular outer measure iff $v^{0}=v$.

We note that $\mu^{\prime}$ is regular if $\mu \in I(L)$. Also, if $\mu \in I_{\sigma}(L)$ then $\mu^{\prime \prime}$ is regular. We have:

PROPOSITION 4.1. Let $X \neq \varnothing$ be a set, $v$ a finite, f.s.a. outer measure.

(a) If $v$ is cover regular, then $v$ is regular.

(b) If $v$ is regular, then $E \in S_{v}$ iff $v(X)=v(E)+v\left(E^{\prime}\right)$.

PROOF.

(a) This follows from a standard greatest lower bound argument, and the monotonicity of $v$.

(b) The proof is similar to that in standard measure theory with a mild $\varepsilon$ argument at the end.

This completes the proof.

We now apply Proposition 4.1 to obtain:

THEOREM 4.1. If $\delta\left(L^{\prime}\right)$ separates $L$, and $\mu \in M_{w}(L) \cap M_{\mathrm{o}}\left(L^{\prime}\right)$, then $\mu \in M_{R}(L)$.

PROOF. To show that $\mu \in M_{R}(L)$ it suffices to show that $L \subset S_{\mu^{\prime}}$.

Let $L \in \mathcal{L}$, and let $\varepsilon>0$ be given and arbitrary. Since $\mu \in M_{w}(L)$, there exists $K_{0} \in \mathcal{L}$ s.t. $K_{0} \subset L^{\prime}$ and

(1) $\mu\left(L^{\prime}\right)-\frac{\varepsilon}{2}<\mu^{\prime}\left(K_{0}\right) \leq \mu\left(L^{\prime}\right)$.

Since $K_{0} \cap L=\varnothing$, and $\delta\left(L^{\prime}\right)$ separates $L$, there exists $U, V \in \delta\left(L^{\prime}\right)$ s.t.

$$
U=\bigcap_{n-1}^{\infty} U_{n}^{\prime}, \quad V=\bigcap_{n=1}^{\infty} V_{n}^{\prime}, \quad K_{0} \subset U, L \subset V, \text { and } U \cap V=\bigcap_{n=1}^{\infty}\left(U_{n}^{\prime} \cap V_{n}^{\prime}\right)=\varnothing \text {, }
$$

and we may assume that $U^{\prime} \cap V_{n}^{\prime} \downarrow \varnothing$.

Since $\mu \in M_{\mathrm{o}}\left(L^{\prime}\right)$, the choice of the sequence $\left\{U_{n}^{\prime} \cap V_{n}^{\prime}\right\}$ from $L^{\prime}$ requires that $\mu\left(U^{\prime}{ }_{n} \cap V_{n}^{\prime}\right) \rightarrow 0$. Therefore, there exists $N \in N$ s.t. $\mu\left(U_{n}^{\prime} \cap V_{n}^{\prime}\right)<\frac{8}{2}$ for $n \geq N$.

$$
\begin{aligned}
\text { Now, } \mu\left(U_{n}^{\prime} \cap V_{n}^{\prime}\right)=\mu\left(U_{n}^{\prime}\right)+\mu\left(V_{n}^{\prime}\right)-\mu\left(U_{n}^{\prime} \cap V_{n}^{\prime}\right) \text { for } n=1,2, \ldots . \text { Thus, if } n \geq N, \\
\mu\left(U_{n}^{\prime} \cup V_{n}^{\prime}\right) \geq \mu\left(U_{n}^{\prime}\right)+\mu\left(V_{n}^{\prime}\right)-\frac{\varepsilon}{2} \\
\Rightarrow \mu\left(U_{n}^{\prime} \cup V_{n}^{\prime}\right) \geq \mu^{\prime}\left(K_{0}\right)+\mu^{\prime}(L)-\frac{\varepsilon}{2} \\
\Rightarrow \mu\left(U_{n}^{\prime} \cup V_{n}^{\prime}\right) \geq \mu\left(L^{\prime}\right)+\mu^{\prime}(L)-\varepsilon \quad \text { by }(1) .
\end{aligned}
$$


Therefore, $\mu\left(U_{n}^{\prime} \cup V_{n}^{\prime}\right) \geq \mu^{\prime}\left(L^{\prime}\right)+\mu^{\prime}(L)-\varepsilon$ since $\mu=\mu^{\prime}$ on $L^{\prime}$. Therefore, $\mu(X)=\mu^{\prime}(X) \geq \mu\left(U_{n}^{\prime} \cup V_{n}^{\prime}\right) \geq \mu^{\prime}\left(L^{\prime}\right)+\mu^{\prime}(L)$. Hence, $L \in S_{\mu^{\prime}} \Rightarrow \mathcal{L} \subset S_{\mu^{\prime}}$. Therefore, $\mu \in M_{R}(L)$.

The proof is now complete.

THEOREM 4.2. Suppose $\mu \in M_{\mathrm{o}}(\Omega)$.

(a) If $\mu=\mu^{\prime \prime}$ on $\mathcal{L}$, and $\mu^{\prime \prime}$ is regular, then $\mathcal{L} \subset S_{\mu^{\prime \prime}}$.

(b) If $\mu=\mu^{\prime \prime}$ on $L$, and $\mu^{\prime \prime}$ is regular, then $\mu \in M^{\circ}(L)$.

(c) If $\mu=\mu^{\prime \prime}$ on $\mathcal{L}$, and $\mu^{\prime \prime}$ is regular, and $\mathcal{L}$ s.s. $\delta(\mathcal{L})$, then $\mu \in M_{R}^{\mathrm{o}}(\mathcal{L})$.

PROOF.

(a) Let $L \in \mathcal{L}$ be arbitrary.

(1) $\mu(X)=\mu(L)+\mu\left(L^{\prime}\right)$.

Since $\mu=\mu^{\prime \prime}$ on $L, \mu(X)=\mu^{\prime \prime}(X), \mu(L)=\mu^{\prime \prime}(L)$, and by definition of $\mu^{\prime \prime}$, i.e., $\mu^{\prime \prime}<\mu^{\prime}$,

(2) $\mu^{\prime \prime}\left(L^{\prime}\right) \leq \mu^{\prime}\left(L^{\prime}\right)=\mu\left(L^{\prime}\right)$, since $\mu-\mu^{\prime}$ on $L^{\prime}$.

It follows from (1) and (2) that

(3) $\mu^{\prime \prime}(X)>\mu^{\prime \prime}(L)+\mu^{\prime \prime}\left(L^{\prime}\right)$.

Hence clearly $L \in S_{\mu^{\prime \prime}}$ since $\mu^{\prime \prime}$ is regular. Therefore, $L \subset S_{\mu^{\prime \prime}} \Rightarrow \mathcal{A}(L) \subset S_{\mu^{\prime \prime}}$.

(b) Since $\mu-\mu^{\prime \prime}$ on $\mathcal{L}$, and $\mu^{\prime \prime}$ is countably additive on $\mathcal{A}(\mathcal{L})$, it follows that $\mu \in M^{\mathrm{\sigma}}(\mathcal{L})$.

(c) Let $L \in \mathcal{L}$ and let $\varepsilon>0$ be given and arbitrary. By definition of $\mu^{\prime \prime}$, there exists a sequence $\left\{L_{n}\right\}$ from $\mathcal{L}$ s.t. $L \subset \bigcup_{n=1}^{\infty} L_{n}^{\prime}$ and

(1) $\sum_{n-1}^{\infty} \mu\left(L_{n}^{\prime}\right)<\mu^{\prime \prime}(L)+\varepsilon$ since $\mu=\mu^{\prime \prime}$ on $L$.

Since $\mu^{\prime \prime}<\mu^{\prime}$ and $\mu^{\prime}=\mu$ on $L^{\prime}, \mu^{\prime \prime}\left(L_{n}^{\prime}\right) \leq \mu\left(L_{n}^{\prime}\right)$ for $n=1,2, \ldots$.

(2) Therefore, $\sum_{n=1}^{\infty} \mu^{\prime \prime}\left(L_{n}^{\prime}\right) \leq \sum_{n=1}^{\infty} \mu\left(L_{n}^{\prime}\right)$.

The countable subadditivity of $\mu^{\prime \prime}$ gives:

(3) $\mu^{\prime \prime}\left(\bigcup_{n=1}^{\infty} L_{n}^{\prime}\right) \leq \sum_{n=1}^{\infty} \mu^{\prime \prime}\left(L_{n}^{\prime}\right)$.

Combining (1), (2), (3) we obtain

(4) $\mu^{\prime \prime}\left(\bigcup_{n=1}^{\infty} L^{\prime}{ }_{n}\right)<\mu(L)+\varepsilon$

Since $\mathcal{L} \subset S_{\mu^{\prime \prime}}$ by (a), it follows from (4) that

(5) $\mu^{\prime \prime}\left(\bigcap_{n-1}^{\infty} L_{n}\right)>\mu\left(L^{\prime}\right)-\varepsilon \geq \mu^{\prime \prime}\left(L^{\prime}\right)-\varepsilon$, where $L^{\prime} \supset \bigcap_{n=1}^{\infty} L_{n}, L_{n} \in \mathcal{L}, n=1,2, \ldots$

Since $\varepsilon>0$ was arbitrary, we conclude from (5) that

(*) $\mu^{\prime \prime}\left(L^{\prime}\right)=\sup \left\{\mu^{\prime \prime}\left(\bigcap_{n=1}^{\infty} L_{n}\right) \mid L^{\prime} \supset \bigcap_{n=1}^{\infty} L_{n}, L_{n} \in \mathcal{L}, n=1,2, \ldots\right\}$ for any $L \in \mathcal{L}$.

By (b), $\mu \in M^{\text {o }}(\mathcal{L})$. Therefore, since $L S_{1} S_{1} S(L)$ it follows by $\left(^{*}\right)$ that $\mu-\mu^{\prime \prime} \operatorname{lac}(c)$ where $\mu^{\prime \prime} \in M_{R}^{\text {o }}(L)$.

The proof is now complete.

REMARK.

(c) is true in particular if $\mu=\mu^{\prime \prime}$ on $\mathcal{L}$ and $\mu^{\prime \prime}$ is regular and $\mathcal{L}$ is a $\delta$-Lattice.

THEOREM 4.3. If $\mu \in J(L)$ and $L \subset S_{\mu^{\prime \prime}}$, then $\mu \in M^{\sigma}(L)$.

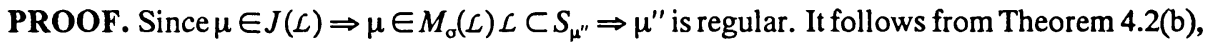
that it suffices to show that $\mu=\mu^{\prime \prime}$ on $\mathcal{L}$. 
(1) Now since $\mu \in J(L), \mu-\mu^{\prime}-\mu^{\prime \prime}$ on $L$. We always have $\mu \leq \mu^{\prime \prime}$ on $L$.

If there exists an $L \in \mathcal{L}$ s.t. $\mu(L)<\mu^{\prime \prime}(L)$, then since $L \subset S_{\mu^{\prime \prime}} \mu\left(L^{\prime}\right)>\mu^{\prime \prime}\left(L^{\prime}\right)-\mu\left(L^{\prime}\right)$ by (1), a contradiction.

Therefore, it must be the case that $\mu=\mu^{\prime \prime}$ on $L$. We conclude that $\mu \in M^{\circ}(L)$.

The proof is now complete.

Note:

(1) If $\mu^{\prime}=\mu^{\prime \prime}$ on $\mathcal{L}^{\prime}$, and if $\mu^{\prime \prime}$ is regular then $\mu \in J(L)$. (This result can be found in [5].)

(2) If $\mu \in J(L)$ then $\mu^{\prime}=\mu^{\prime \prime}$ on $L^{\prime}$.

We conclude by extending a result in [5]; namely,

THEOREM 4.4.

(a) If $\bigcup_{n=1}^{\infty} L^{\prime}{ }_{n} \in L^{\prime}$ where $L_{n} \in \mathcal{L}$ for all $n$ and if $\mu \in J(L)$, then $\mu\left(\bigcup_{n=1}^{\infty} L^{\prime}{ }_{n}\right) \leq \sum_{n=1}^{\infty} \mu\left(L_{n}^{\prime}\right)$.

(b) If $\mu\left(\bigcup_{n=1}^{\infty} L_{n}^{\prime}\right) \leq \sum_{n=1}^{\infty} \mu\left(L_{n}^{\prime}\right)$ whenever $\bigcup_{n=1}^{\infty} L^{\prime}{ }_{n} \in \mathcal{L}$ for all $n$, and if $\mu^{\prime \prime}$ is regular, then $\mu \in J(L)$.

PROOF.

(a) See [5].

(b) We know that $\mu^{\prime \prime} \leq \mu$ on $L^{\prime}$. Suppose there exists $L^{\prime} \in L^{\prime}$ s.t. $\mu^{\prime \prime}\left(L^{\prime}\right)<\mu\left(L^{\prime}\right)$. Then there exists $L_{n} \in \mathcal{L}$ s.t. $L^{\prime} \subset \bigcup_{n=1}^{\infty} L_{n}^{\prime}$ and $\sum_{n=1}^{\infty} \mu\left(L^{\prime}{ }_{n}\right)<\mu\left(L^{\prime}\right)$.

But $L^{\prime}=\bigcup_{n=1}^{\infty}\left(L^{\prime}{ }_{n} \cap L^{\prime}\right)$. Therefore, $\mu\left(L^{\prime}\right)=\mu\left(\bigcup_{n=1}^{\infty}\left(L^{\prime} \cap L^{\prime}\right)\right) \leq \sum_{n=1}^{\infty} \mu\left(L^{\prime}{ }_{n} \cap L^{\prime}\right)$ by hypothesis. Therefore, $\mu\left(L^{\prime}\right) \leq \sum_{n=1}^{\infty} \mu\left(L_{n}^{\prime}\right)<\mu\left(L^{\prime}\right)$ which is a contradiction. Therefore, $\mu^{\prime \prime}-\mu^{\prime}$ on $L^{\prime}$, and since $\mu^{\prime \prime}$ is regular it follows easily that $\mu \in J(L)$.

The proof is now complete.

ACKNOWLEDGMENT. The author wishes to thank the referee for suggestions which improved the abstract, and the presentation of several statements.

\section{REFERENCES}

[1] BACHMAN, G. and STRATIGOS, P., On general lattice repleteness and completeness, Illinois Jour. of Math. 27, no. 4 (1993), 535-561.

[2] CAMACHO, J. Jr., Extension of lattice regular measures with applications, Jour. Indian Math. Soc. 54 (1989), 233-244.

[3] FROLIK, Z., Prime filters with the c.i.p., Comm. Math. Univ. Carolina 13 (1972), 553-575.

[4] GILLMAN, L. and JERISON, M., Rings of Continuous Functions, Van Nostrand, Princeton, NJ, 1960.

[5] GRASSI, P., Outer measures and associated lattice properties, Internat. J. Math. and Math. Sci. 16, no. 4 (1993), 687-694.

[6] SIEGEL, D., Outer measures and weak regularity of measures (to appear Internat. J. Math. and Math. Sci.).

[7] SZETO, M., On normal lattices and separation properties of lattices, Jour. Indian Math. Soc., 58, no. 1 (1992), 51-64.

[8] SZETO, M., On separation of lattices, Internat. J. Math. and Math. Sci., 14, no. 2 (1991), 325-338.

[9] WALLMAN, H., Lattices and topological spaces, Ann. of Math. 39 (1938), 112-126. 


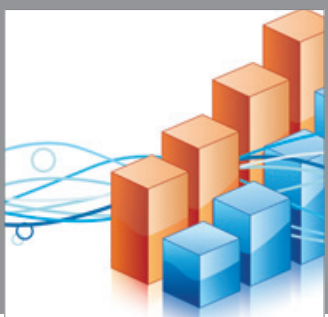

Advances in

Operations Research

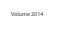

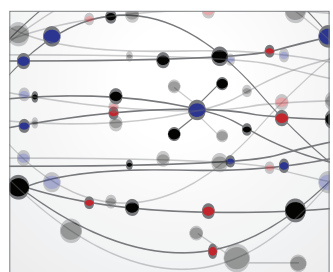

\section{The Scientific} World Journal
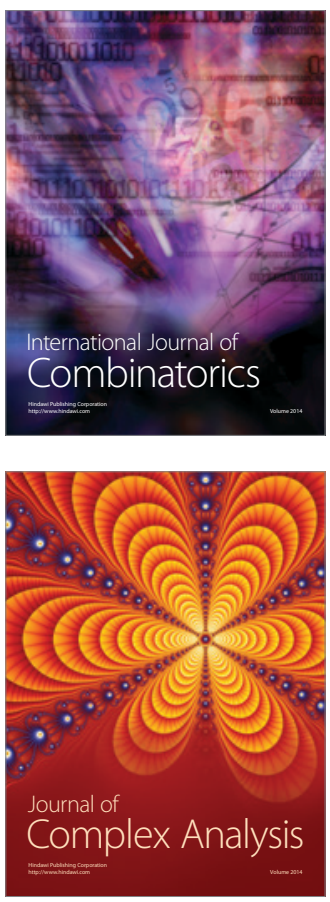

International Journal of

Mathematics and

Mathematical

Sciences
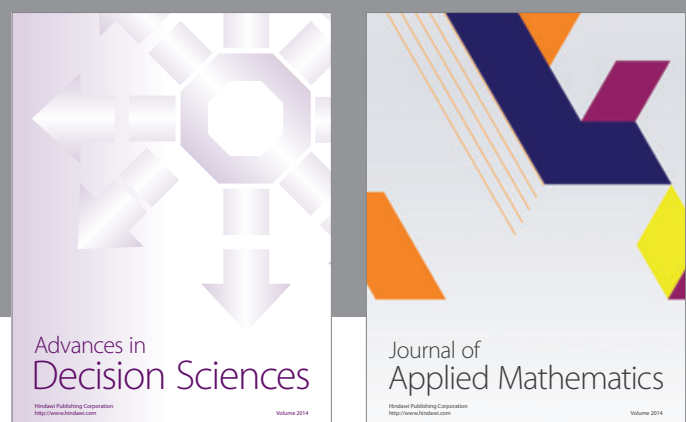

Journal of

Applied Mathematics
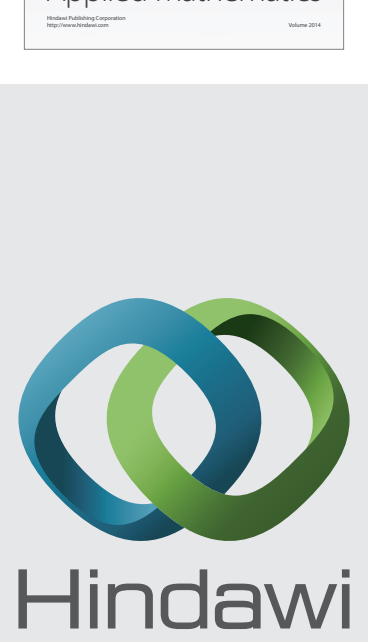

Submit your manuscripts at http://www.hindawi.com
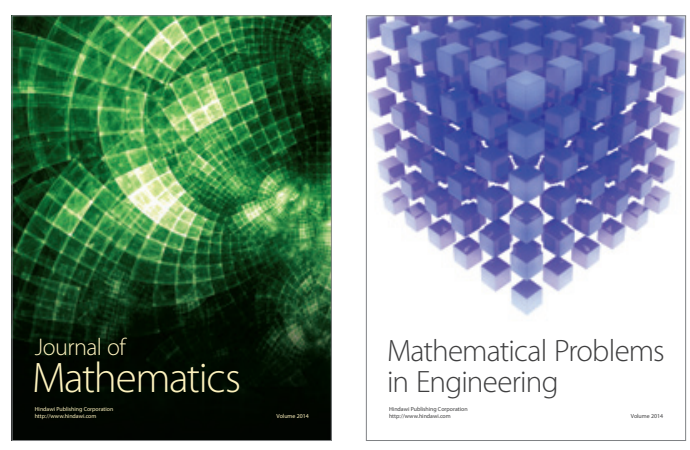

Mathematical Problems in Engineering
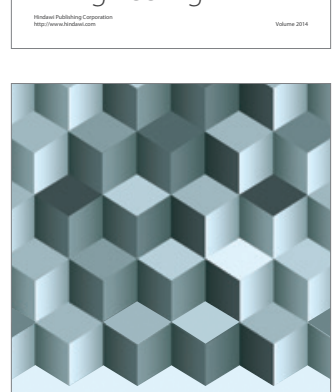

Journal of

Function Spaces
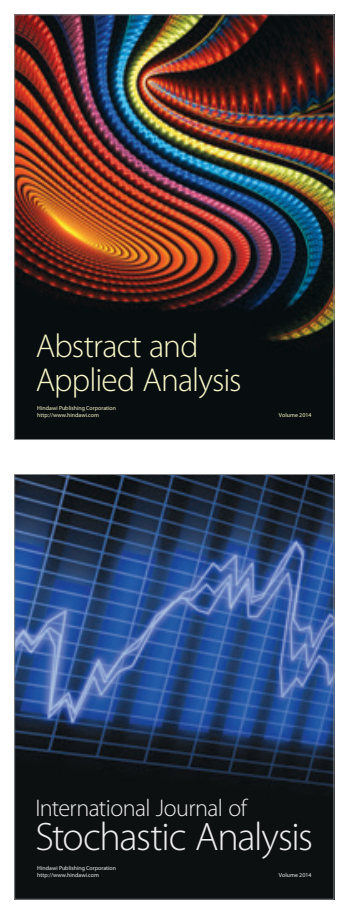

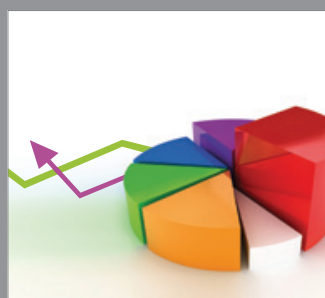

ournal of

Probability and Statistics

Promensencen
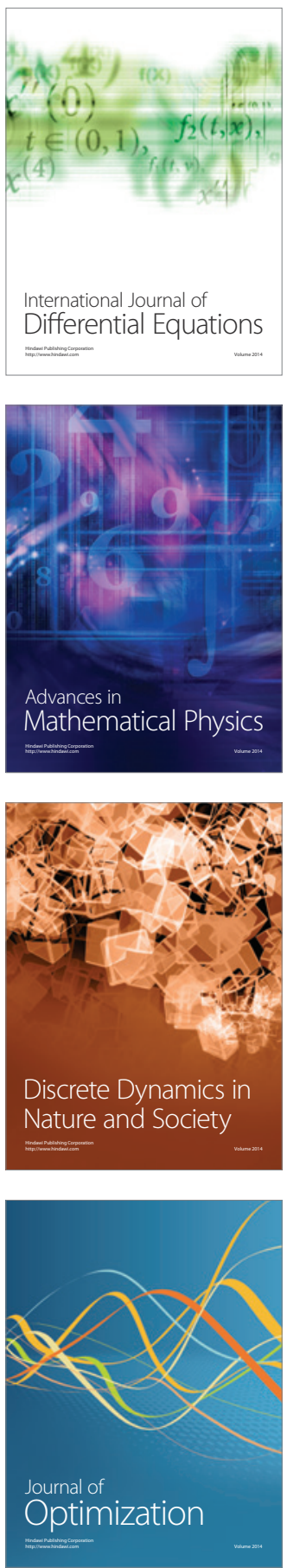